\title{
Estrategias de gestión de tesorería en las pequeñas y medianas empresas del sector petroquímico
}

\author{
Treasury management strategies in small and medium-sized enterprises in the \\ petrochemical sector
}

\begin{tabular}{l|r}
\cline { 2 - 2 } & Emilio Díaz \\
\hline $\begin{array}{l}\text { Artículo recibido en mayo } 2019 \\
\text { Arbitrado en junio 2019 } \\
\text { Publicado en septiembre } 2019\end{array}$ & emiliodiaz3@gmail.com \\
& ORCID: 0000-0001-9528-1578 \\
Universidad del Zulia - Venezuela
\end{tabular}

RESUMEN

Palabras clave:

ABSTRACT

Keywords:
La investigación tuvo como objetivo analizar las estrategias de gestión de tesorería en las pequeñas y medianas empresas del sector petroquímico. La misma fue sustentada con una metodología de tipo descriptiva, bajo un diseño no experimental, transeccional y de campo. La población quedo constituida por seis empresas, vigentes y en ejecución dentro del complejo petroquímico del municipio Miranda. Para la recolección de la información se utilizó un instrumento, tipo cuestionario, conformado por trece ítems, con cinco opciones de respuestas. Para su validez se sometió al juicio de expertos. Para la confiabilidad, se empleó el Método Alfa de Cronbach, obteniéndose 0,96. Se tabularon los datos procediendo al análisis cuantitativo de frecuencias absolutas y relativas. Se resalta que los indicadores: flujo de cobros, flujo de pagos, posición fecha valor, previsiones de tesorería y funciones del tesorero se posicionaron en muy alta aplicación; sólo los depósitos a plazo fijo ostentaron alta aplicación.

Depósitos a plazo fijo, estrategias de gestión de tesorería, flujo de cobros, flujo de pagos, funciones del tesorero, posición fecha valor, previsiones de tesorería

The research aimed to analyze treasury management strategies in small and mediumsized companies in the petrochemical sector. It was supported by a descriptive methodology, under a non-experimental, transectional and field design. The population was made up of six companies, in force and in execution within the petrochemical complex of the Miranda municipality. To collect the information, an instrument, questionnaire type, consisting of thirteen items, with five response options was used. For its validity, it was submitted to expert judgment. For reliability, the Cronbach's Alpha Method was used, obtaining 0.96. The data were tabulated proceeding to the quantitative analysis of absolute and relative frequencies. It is highlighted that the indicators: collection flow, payment flow, value date position, treasury forecasts and treasurer functions were positioned in very high application; only fixed-term deposits held high application.

Time deposits, cash management strategies, cash flow, cash flow, treasurer's functions, value date position, cash forecast 


\section{INTRODUCCIÓN}

Las estrategias financieras $y$ empresariales deben ser coherentes entre sí y apoyarse mutuamente. Deben trabajar juntas y no estar en conflicto. El logro de tal integración o coherencia tiene efectos positivos en la ejecución de estrategias, tanto en el nivel financiero como en el empresarial. La necesidad de coherencia y equilibrio entre las estrategias financieras y empresariales es una clara condición sugerida por el análisis precedente en cuanto al planeamiento sólido. La revisión de la estrategia es otro paso importante en la integración de las estrategias de los niveles financiero y empresarial, ayuda a fomentar el análisis, comunicación y debate entre los niveles $y$, garantiza que la organización ejecute buenos planes.

En este orden de ideas, considerando las pequeñas y medianas empresas del sector petroquímico del municipio Miranda, las cuales son objeto de estudio de la presente investigación, y se encargan de prestar servicios específicos en ese sector económico, deben igualmente implementar en sus finanzas las estrategias financieras acordes al tipo de negocio. Al respecto, cabe resaltar que en Venezuela las Pymes que prestan servicio a la industria nacional, se desenvuelven en una serie de problemas que en la mayoría de los casos no logran superar; lo que conlleva hacia el fracaso seguro que tiende a complicarse bajo la presencia de ciertos elementos que obstaculizan no solo su crecimiento sino también su desarrollo; por lo cual deben estar en evaluación continua de todas sus estrategias, para este caso en específico, las estrategias financieras.

Desde este punto de vista, la formulación, así como la ejecución, de acertadas estrategias financieras influyen en cualquier tipo de organización, siendo las pequeñas y medianas empresas aquellas donde se siente con mayor intensidad, y quienes asumen la postura de negar la existencia e influencia de éstas, tienden al fracaso empresarial.

Visto así, la adopción de estrategias financieras, en el entorno empresarial tan complejo como el que vive Venezuela, va más allá de la de ser una necesidad para el crecimiento empresarial, en relación a ello Fernández (2006, p. 30) afirma "se ha transformado en una prioridad para la alta dirección, ligada a la propia supervivencia de la compañía". De allí que los empresarios, los diferentes grupos de accionistas, los inversionistas, hasta los reguladores, apuestan ahora más que nunca por el fortalecimiento de los sistemas de gestión y control financiero, que les permita no solo generar armonía, sino también preservar valor para mantener un desarrollo sostenible en el tiempo.

En particular, según la información obtenida por el investigador, a través de entrevistas informales a los propietarios, muchas de la Pymes arraigadas en la Costa Oriental de Lago, específicamente en el Municipio Miranda, prestadoras de servicios a la industria petroquímica nacional y sus filiares, no escapan de la realidad vivida en el país. En esta tónica, es importante resaltar que la mayoría de estas empresas, por lo general, son empresas familiares que operan en mercado locales, y en su mayoría desconocen la relevancia de la planificación así como de la formulación de estrategias financieras y como gestionarlas.

Del mismo modo, poseen escaso talento humano calificado, lo que se traduce en un bajo grado de profesionalización, mala 
identificación de los factores tanto interno como externos, lo cual incide severamente en su desempeño al momento de gestionar las decisiones en materia de asumir nuevos proyecto de inversión. Asimismo, se conoció que estas empresas se han visto afectadas en lo económico dado que, en su mayoría, prestan servicios a las petroquímicas, las cuales dependen de los recursos del Estado para cubrir sus compromisos y, como es del conocimiento de todos, con la situación económica que actualmente presenta el país estos compromisos demoran hasta más de tres meses luego de su vencimiento.

Esta es la razón fundamental por la que se desea analizar las estrategias de gestión de tesorería en las pequeñas y medianas empresas del sector petroquímico, para que éstas puedan conocer mecanismos empresariales que les permitan enfrentar las condiciones de incertidumbre por las que normalmente transitan, brindándoles aportes que contribuyan, de alguna forma, a orientar la administración de sus finanzas, y por ende, el incremento de su rentabilidad.

\section{MATERIALES Y METODO}

La metodología aplicada es de tipo descriptiva, con un diseño no experimental, transeccional y de campo. La población quedo constituida por seis empresas, vigentes $y$ en ejecución dentro del complejo petroquímico Ana María Campo: Consermantimec, Simnco, Socoven, Servicios Técnico Mecánicos, Aistercon, y Cavem, en el municipio Miranda. Las unidades informantes fueron los gerentes, coordinadores y líderes (de acuerdo a sus estructuras organizativas) encargados del área financiera, estimándose un total de veintidós (22) personas.

Para la recolección de la información se utilizó un instrumento, tipo cuestionario, conformado por trece ítems, con cinco opciones de respuestas bajo la escala de frecuencia. Para su validez se sometió al juicio de cinco expertos. Para la confiabilidad, se empleó el Método Alfa de Cronbach, cuyo valor fue 0,96 .

Se tabularon los datos procediendo al análisis cuantitativo de frecuencias absolutas y relativas. A tales efectos, para el análisis de las frecuencias el investigador consideró conveniente fijar un baremo de interpretación, el cual se recoge en el cuadro 1 , en el mismo se muestra el rango en donde pueden darse los resultados con base a la suma de las respuestas más positivas, siempre y casi siempre $(\mathrm{S}+\mathrm{Cs})$, la categoría asignada, así como la descripción de la misma.

Tabla 1. Interpretación del porcentaje de la distribución de frecuencia

\begin{tabular}{ccl}
\hline Rango & Categoría & \multicolumn{1}{c}{ Interpretación } \\
\hline $\mathrm{S}+\mathrm{CS} \geq 70 \%$ & $\begin{array}{c}\text { Alta aplicación } \\
\text { Alta fortaleza }\end{array}$ & $\begin{array}{l}\text { Alta aplicación del indicador, dimensión o variable. } \\
\text { Implica alta fortaleza para las empresas }\end{array}$ \\
$40 \% \leq \mathrm{S}+\mathrm{CS}<70 \%$ & $\begin{array}{c}\text { Moderada } \\
\text { aplicación } \\
\text { Leve fortaleza }\end{array}$ & $\begin{array}{l}\text { Moderada aplicación del indicador, dimensión o } \\
\text { variable. Implica leve fortaleza para las empresas }\end{array}$ \\
$\mathrm{S}+\mathrm{CS}<40 \%$ & $\begin{array}{c}\text { Baja aplicación } \\
\text { Debilidad }\end{array}$ & $\begin{array}{l}\text { Baja aplicación del indicador, dimensión o variable. } \\
\text { Implica debilidad para las empresas }\end{array}$ \\
\hline
\end{tabular}

Fuente: Díaz (2020) 
RESULTADOS Y DISCUSION

Como se puede observar, en la tabla 2 se muestra el comportamiento del indicador flujo de cobros, de la dimensión estrategias de gestión de tesorería; observándose un promedio de frecuencias de $97,73 \%$, indicando que siempre y casi siempre, a criterio de los encuestados, el flujo de cobros, como parte de las estrategias de gestión de tesorería que utilizan, se aplica con muy alta frecuencia, otorgándole la categoría de muy alta aplicación, implicando a su vez una alta fortaleza para dichas empresas.

Tabla 2. Indicador: Flujo de cobros

\begin{tabular}{|c|c|c|c|c|}
\hline \multicolumn{5}{|l|}{ Ítems del indicador } \\
\hline \multicolumn{5}{|c|}{ 1. Consideran el uso del flujo de cobros en la gestión de la tesorería. } \\
\hline \multicolumn{5}{|c|}{$\begin{array}{l}\text { 2. Se hace seguimiento continuo al proceso de cobros para que el efectivo sea } \\
\text { esperado. }\end{array}$} \\
\hline \multirow{2}{*}{ Alternativas } & \multicolumn{2}{|c|}{17} & \multicolumn{2}{|c|}{18} \\
\hline & FA & FR (\%) & FA & FR (\%) \\
\hline Siempre & 18 & 81,82 & 12 & 54,55 \\
\hline Casi siempre & 3 & 13,64 & 10 & 45,45 \\
\hline Algunas veces & 1 & 4,55 & 0 & 0,00 \\
\hline Casi nunca & 0 & 0,00 & 0 & 0,00 \\
\hline \multirow[t]{2}{*}{ Nunca } & 0 & 0,00 & 0 & 0,00 \\
\hline & 22 & 100 & 22 & 100 \\
\hline \multirow[t]{2}{*}{$S+C s$} & \multicolumn{2}{|c|}{$95,45 \%$} & \multicolumn{2}{|c|}{$100 \%$} \\
\hline & \multicolumn{2}{|c|}{ Muy alta aplicación } & \multicolumn{2}{|c|}{ Muy alta aplicación } \\
\hline Categoría & \multicolumn{2}{|c|}{ Alta fortaleza } & \multicolumn{2}{|c|}{ Alta fortaleza } \\
\hline \multicolumn{3}{|l|}{ Promedio Indicador } & \multicolumn{2}{|c|}{$97,73 \%$} \\
\hline Categoría & \multicolumn{4}{|c|}{ Muy alta aplicación / Alta fortaleza } \\
\hline
\end{tabular}

Fuente: Díaz (2020)

Estos resultados son reflejo de las actividades que se desarrollan en las empresas bajo estudio. En este sentido al consultarles si consideran el uso del flujo de cobros en la gestión de la tesorería y, hacen seguimiento continuo al proceso de cobros para que el efectivo sea percibido en el momento esperado, un $95,45 \%$ y un $100 \%$ consideró las opciones más positivas ( $\mathrm{S}+$ Cs) catalogadas con muy alta aplicación e implicando altas fortalezas, según el baremo utilizado.
Los resultados validan lo postulado por Santoma (2008), para quien el flujo de cobros de la empresa es una función vital para la buena marcha de todo negocio, ya que es el que aporta el disponible necesario para que la empresa pueda hacer frente a sus propias obligaciones. Asimismo, es la fase donde la empresa realiza sus beneficios, puesto que hasta que la venta no está cobrada no se puede considerar cerrada la transacción comercial. 
A criterio del investigador, dado los resultados expuestos, las empresas en estudio conciben el flujo de cobros como una función estratégica de su gestión de tesorería, entendiendo que su proceso de cobro es el generador de liquidez para la empresa y lo que permite su buen funcionamiento. Adicionalmente, consideran el factor tiempo como algo esencial del circuito de cobros, asimismo deben considerar cerrar el ciclo cuando se disponga plenamente del importe de la venta en la tesorería de la empresa.
El siguiente indicador que se analiza, en esta dimensión, es el denominado: flujo de pagos. Los resultados relativos a él, se resumen en la tabla 3 , observándose que, en promedio, para el $97,73 \%$ de los encuestados al flujo de pagos, siempre y casi siempre, se le otorga muy alta aplicación como estrategias de gestión de tesorería, implicando alta fortaleza de la gestión financiera que llevan a cabo las empresas bajo estudio.

Tabla 3. Indicador: Flujo de pagos

\begin{tabular}{|c|c|c|c|c|c|}
\hline \multicolumn{6}{|c|}{ Ítems del indicador } \\
\hline 1. & \multicolumn{5}{|c|}{ Se realizan los pagos de manera oportuna. } \\
\hline 2. & \multicolumn{5}{|c|}{ Se consideran los descuentos por pronto pago. } \\
\hline \multirow{2}{*}{\multicolumn{2}{|c|}{ Alternativas }} & \multicolumn{2}{|c|}{19} & \multicolumn{2}{|c|}{20} \\
\hline & & FA & FR (\%) & FA & \\
\hline \multicolumn{2}{|c|}{ Siempre } & 6 & 27,27 & 2 & 9,09 \\
\hline \multicolumn{2}{|c|}{ Casi siempre } & 15 & 68,18 & 20 & 90,91 \\
\hline \multicolumn{2}{|c|}{ Algunas veces } & 1 & 4,55 & 0 & 0,00 \\
\hline \multicolumn{2}{|c|}{ Casi nunca } & 0 & 0,00 & 0 & 0,00 \\
\hline \multicolumn{2}{|c|}{ Nunca } & 0 & 0,00 & 0 & 0,00 \\
\hline & Total & 22 & 100 & 22 & 100 \\
\hline \multicolumn{2}{|c|}{$S+C s$} & \multicolumn{2}{|c|}{$95,45 \%$} & \multicolumn{2}{|c|}{$100 \%$} \\
\hline \multicolumn{2}{|c|}{ Categoría } & \multicolumn{2}{|c|}{$\begin{array}{l}\text { Muy alta aplicación } \\
\text { Alta fortaleza }\end{array}$} & \multicolumn{2}{|c|}{$\begin{array}{l}\text { Muy alta aplicación } \\
\text { Alta fortaleza }\end{array}$} \\
\hline \multicolumn{2}{|c|}{ Promedio Indicador } & \multicolumn{4}{|c|}{$97,73 \%$} \\
\hline \multicolumn{2}{|c|}{ Categoría } & \multicolumn{4}{|c|}{ Muy alta aplicación / Alta fortaleza } \\
\hline
\end{tabular}

Fuente: Díaz (2020)

Adicionalmente, la tabla muestra como para el $100 \%$ de los encuestados, siempre y casi siempre se consideran los descuentos por pronto pago, y el $95,45 \%$ opinó que siempre y casi siempre se realizan los pagos de manera oportuna, asignándole a estas actividades la categoría de muy alta aplicación, constituyéndolas en altas fortalezas para las empresas estudiadas.

Los resultados alcanzados logran validar, de manera alta, lo planteado por Brachfield (2009), para quien el objetivo de cualquier tesorero ha de ser pagar siempre en el momento que fue establecido, cuando se pactó este asunto y, en la 
manera oportuna. $\mathrm{Ni}$ un minuto antes, pues se perdería lógicamente rentabilidad; ni por supuesto, un segundo después, ya que la imagen que el acreedor tendría de la empresa se vería dañada por este preciso motivo y esto es, sin lugar a dudas, algo bastante nocivo para el objetivo estratégico que tiene toda empresa.

Adicional a esto, también validan al investigador, quien sugiere que una buena labor del tesorero en lo relativo al flujo de pagos, debe ser la realización de todo lo necesario para que los pagos a realizar sean hechos efectivos cuanto más antes mejor, sin pensar ni siquiera en el día de pago prefijado a fin de respetar los compromisos dados.

Siguiendo el análisis de resultados se muestra la tabla 4 la cual recoge los hallazgos obtenidos para el indicador posición fecha valor. Como se puede apreciar el 93,18\% de los encuestados, en promedio, opinan que la posición fecha valor como estrategia de tesorería posee muy alta aplicación en las empresas bajo estudio, confiriéndoles a éstas una alta fortaleza al respecto.
Estos resultados obedecen a la categoría dada a los ítems utilizados, donde se evidencia que un $95 \%$ opina que siempre y casi siempre emplean alguna estrategia para realizar previsiones según la fecha-valor de sus saldos bancarios, y el $91 \%$ declara que a nivel de tesorería, se toma como referencia el saldo bancario según su fecha-valor; confiriéndole en ambos casos altas fortalezas a las empresas estudiadas en lo concerniente a estos aspectos.

Los resultados muestran muy alta congruencia con lo expuesto por Olsina (2009), quien afirma que debe considerarse la fecha-valor, tanto para realizar las previsiones de saldos bancarios como para negociar con los bancos su minimización. Para una eficiente gestión de tesorería es imprescindible tomar como referencia el saldo en fecha-valor y no el saldo contable. Este autor establece que no es arriesgado decir que el concepto más importante en la gestión de tesorería es la fecha valor. 
Tabla 4. Indicador: Posición fecha valor

\section{Ítems del indicador}

3. Emplean alguna estrategia para realizar previsiones según la fecha-valor de sus saldos bancarios.

4. A nivel de tesorería, se toma como referencia el saldo bancario según su fecha-valor.

\begin{tabular}{|c|c|c|c|c|c|}
\hline & \multicolumn{2}{|c|}{21} & \multicolumn{2}{|c|}{22} \\
\hline & & FA & FR (\%) & FA & FR (\%) \\
\hline \multicolumn{2}{|l|}{$\begin{array}{l}\text { Alternativas } \\
\text { Siempre }\end{array}$} & 5 & 22,73 & 18 & 81,82 \\
\hline \multicolumn{2}{|l|}{ Casi siempre } & 16 & 72,73 & 2 & 9,09 \\
\hline \multicolumn{2}{|l|}{ Algunas veces } & 0 & 0,00 & 2 & 9,09 \\
\hline \multicolumn{2}{|l|}{ Casi nunca } & 1 & 4,55 & 0 & 0,00 \\
\hline \multirow[t]{2}{*}{ Nunca } & & 0 & 0,00 & 0 & 0,00 \\
\hline & Total & 22 & 100 & 22 & 100 \\
\hline \multicolumn{2}{|l|}{$S+C s$} & \multicolumn{2}{|c|}{$95 \%$} & \multicolumn{2}{|c|}{$91 \%$} \\
\hline \multicolumn{2}{|l|}{ Categoría } & \multicolumn{2}{|c|}{$\begin{array}{l}\text { Muy alta aplicación } \\
\text { Alta fortaleza }\end{array}$} & \multicolumn{2}{|c|}{$\begin{array}{l}\text { Muy alta aplicación } \\
\text { Alta fortaleza }\end{array}$} \\
\hline \multicolumn{2}{|c|}{$\begin{array}{l}\text { Promedio Indicador } \\
\text { Categoría }\end{array}$} & \multicolumn{4}{|c|}{$\begin{array}{l}93,18 \% \\
\text { Muy alta aplicación / Alta fortaleza }\end{array}$} \\
\hline
\end{tabular}

Fuente: Díaz (2020)

Visto así, para el investigador, las pequeñas y medianas empresas del sector petroquímico del municipio Miranda han logrado fijar una fecha valor en el programa de pagos, así logran obtener la fecha de la salida de fondos, aspecto importante para que la posición de tesorería establezca el número de días de mora. Cabe resaltar que en la elección de sus instrumentos de pago, procuran el que les favorezca a nivel de valoración, tanto en las previsiones de tesorería como en los movimientos bancarios, realizándolos con atención a la fecha valor; esto en las negociaciones bancarias es uno de los aspectos con mayor importancia.
En lo concerniente al indicador depósitos a plazo fijo, se observa, en la tabla 5 , un promedio de frecuencias de $47,73 \%$, indicando baja aplicación de este indicador como estrategia de gestión de tesorería en las empresas analizadas, implicando una leve debilidad, según el baremo diseñado. Así, en el detalle de los ítems, el instrumento diseñado arrojó que el $55 \%$ de los encuestados señalan que, siempre y casi siempre, se le da alta aplicación al que se realicen inversiones en depósitos a plazo fijo como un instrumento generador de intereses (ítem 23) otorgándole leve fortaleza a las empresas bajo estudio, en lo que respecta a este aspecto. 
Tabla 5. Indicador: Depósitos a plazo fijo

\section{Ítems del indicador}

5. Realizan inversiones en depósitos a plazo fijo como un instrumento generador de intereses.

6. Mantienen su rentabilidad financiera a través de depósitos a plazo fijo.

\section{Alternativas}

Siempre

Casi siempre

Algunas veces

Casi nunca

Nunca

Categoría

Promedio Indicador

Categoría

Fuente: Díaz (2020)

Mientras para el ítem 24 el $41 \%$ de los encuestados se posicionaron en las opciones siempre y casi siempre, indicando baja aplicación en lo referido a sí mantienen su rentabilidad financiera a través de depósitos a plazo fijo, concediéndole a las empresas una leve debilidad en este sentido.

Lo mostrado no alcanza a validar, lo postulado por Brachfield (2009), quien opina que las empresas, dentro de su gestión de tesorería, pueden realizar inversiones en depósitos a plazo fijo como un instrumento generador de intereses, también pueden mantener su rentabilidad financiera a través de depósitos a plazo fijo. Pero como no siempre ocurre lo proyectado, es importante tener en cuenta, sobre todo en las pequeñas y medianas empresas, la posible necesidad de liquidez del capital invertido, pues algunos contratos de depósito a plazo permiten la cancelación anticipada mediante una penalización.

En virtud de los resultados, a criterio del investigador, aun cuando se evidencia baja aplicación de los depósitos a plazo fijo en la estrategia de tesorería que llevan a cabo las empresas bajo estudio, ciñéndose al autor citado, es probable que estas empresas quieran mantener liquidez ante las condiciones de inflación que se tiene en Venezuela, obligándolas a contar con dinero a disposición para enfrentar este tipo de flagelo económico, lo cual las lleva a una baja aplicación de los depósitos a plazo fijo.

La tabla 6 muestra los resultados obtenidos para el indicador previsiones de tesorería, observándose un promedio de frecuencias de 97,73\%, indicando que en las empresas estudiadas, siempre y casi siempre, a criterio de los encuestados, las previsiones de tesorería, como parte de las 
estrategias de gestión de tesorería que siguen, se aplica con muy alta frecuencia, otorgándole la categoría de muy alta aplicación, implicando a su vez una alta fortaleza para dichas empresas.

Tabla 6. Indicador: Previsiones de tesorería

Ítems del indicador

7. Resguardan dinero para prever futuras situaciones de liquidez.

8. Poseen un flujo de cobros suficiente para cubrir pagos imprevistos en un horizonte inmediato.

\begin{tabular}{|c|c|c|c|c|}
\hline \multirow{2}{*}{ Alternativas } & \multicolumn{2}{|c|}{25} & \multicolumn{2}{|c|}{26} \\
\hline & FA & FR (\%) & FA & FR (\%) \\
\hline Siempre & 4 & 18,18 & 1 & 4,55 \\
\hline Casi siempre & 18 & 81,82 & 20 & 90,91 \\
\hline Algunas veces & 0 & 0,00 & 1 & 4,55 \\
\hline Casi nunca & 0 & 0,00 & 0 & 0,00 \\
\hline Nunca & 0 & 0,00 & 0 & 0,00 \\
\hline Total & 22 & 100 & 22 & 100 \\
\hline$S+C s$ & \multicolumn{2}{|c|}{$100 \%$} & \multicolumn{2}{|c|}{$95 \%$} \\
\hline Categoría & \multicolumn{2}{|c|}{$\begin{array}{l}\text { Muy alta aplicación } \\
\text { Alta fortaleza }\end{array}$} & \multicolumn{2}{|c|}{$\begin{array}{l}\text { Muy alta aplicación } \\
\text { Alta fortaleza }\end{array}$} \\
\hline $\begin{array}{l}\text { Promedio } \\
\text { Indicador }\end{array}$ & \multicolumn{4}{|c|}{$97,73 \%$} \\
\hline Categoría & \multicolumn{4}{|c|}{ Muy alta aplicación / Alta fortaleza } \\
\hline
\end{tabular}

Fuente: Díaz (2020)

De tal manera, le confirieron muy alta aplicación a las actividades medidas por los ítems 9 y 10, referidas a si resguardan dinero para prever futuras situaciones de liquidez (100\%), y poseen un flujo de cobros suficiente para cubrir pagos imprevistos en un horizonte inmediato (95\%); implicando alta fortaleza para las empresas estudiadas en lo concerniente a estas actividades.

Estos resultados coinciden, de forma muy alta, con lo expuesto por Santoma (2008), quien afirma que dependiendo del uso que la empresa desee hacer de las previsiones será el período de análisis, ya que se pueden realizar previsiones para un horizonte inmediato, anual, superior al año o a largo plazo. Las pequeñas empresas, por lo general realizan la previsión referida a un futuro inmediato, la cual tiene por objeto asegurar que los saldos de tesorería generados por los pagos y los cobros futuros son suficientes para hacer frente a los pagos previstos y, en caso contrario, poder actuar a tiempo mediante la obtención de fondos a corto plazo. Este análisis suele denominarse gestión de la posición de fondos en fecha valor.

A juicio del investigador, con base a estos resultados, en las pequeñas y medianas empresas del sector petroquímico del municipio Miranda se maneja la previsión de tesorería bajo un criterio de previsión a futuro inmediato, asegurándose el resguardar dinero para prever futuras situaciones de liquidez y mantener un flujo 
de cobros suficiente para cubrir pagos imprevistos, situaciones que le otorgan una alta fortaleza en este sentido, según el baremo diseñado. Esta situación reafirma por qué presentan baja aplicación de los depósitos a plazo fijo.

Ahora bien, en el orden de presentación que se lleva adelante, se tienen los resultados del último indicador considerado en la dimensión estrategias de gestión de tesorería, referido a las funciones del tesorero. En este sentido, en la tabla 7, se observa un promedio de frecuencias en las respuestas positivas ( $S+C s$ ) de $100 \%$, indicando muy alta aplicación de este indicador como parte de las estrategias de gestión de tesorería aplicadas por las empresas bajo estudio, lo cual les concede una alta fortaleza.

Al desglosar resultados por ítems, se evidencia para todos muy alta aplicación de las actividades señaladas por ellos, en cuanto a si el tesorero realiza proyecciones sobre los flujos de cobros y sobre los flujos de pago esperados, y si mide el riesgo financiero provocado por las variaciones en los intereses; lo cual les confiere alta fortaleza a las empresas bajo estudio en estos aspectos.

Los resultados validan, en muy alto grado, a Santoma (2008), para quien las funciones del tesorero, por lo general, están en dependencia de la dirección financiera (aunque está muchas veces depende de la función, del tamaño y organización de la misma). En concreto, el tesorero de una empresa debe: decidir los mejores instrumentos de gestión a la administración de la tesorería, hacer proyecciones de flujos monetarios de cobros y pagos de la empresa, llevar control de costes y gastos bancarios, entre otros.

Tabla 7. Indicador: Funciones del tesorero

\section{Ítems del indicador}

9. El tesorero realiza proyecciones sobre los flujos de cobros.

10. El tesorero realiza proyecciones sobre los flujos de pago esperados.

11. El tesorero mide el riesgo financiero provocado por las variaciones en los intereses.

\begin{tabular}{|c|c|c|c|c|c|c|}
\hline \multirow[b]{2}{*}{ Alternativas } & \multicolumn{2}{|c|}{27} & \multicolumn{2}{|c|}{28} & \multicolumn{2}{|c|}{29} \\
\hline & FA & FR (\%) & FA & FR (\%) & FA & FR (\%) \\
\hline Siempre & 18 & 81,82 & 16 & 72,73 & 18 & 81,82 \\
\hline Casi siempre & 4 & 18,18 & 6 & 27,27 & 4 & 18,18 \\
\hline Algunas veces & 0 & 0,00 & 0 & 0,00 & 0 & 0,00 \\
\hline Casi nunca & 0 & 0,00 & 0 & 0,00 & 0 & 0,00 \\
\hline \multirow[t]{2}{*}{ Nunca } & 0 & 0,00 & 0 & 0,00 & 0 & 0,00 \\
\hline & 22 & 100 & 22 & 100 & 22 & 100 \\
\hline $\begin{array}{c}\text { S + Cs } \\
\text { Categoría } \\
\text { Promedio Indicador }\end{array}$ & $\begin{array}{l}\text { Muy a } \\
\text { Alt }\end{array}$ & $\begin{array}{l}\% \\
\text { plicación } \\
\text { aleza }\end{array}$ & \multicolumn{2}{|c|}{$\begin{array}{c}\text { Muy alta aplicación } \\
\text { Alta fortaleza } \\
100 \%\end{array}$} & \multicolumn{2}{|c|}{$\begin{array}{l}\text { Muy alta aplicación } \\
\text { Alta fortaleza }\end{array}$} \\
\hline Categoría & \multicolumn{6}{|c|}{ Muy alta aplicación / Alta fortaleza } \\
\hline
\end{tabular}

Fuente: Díaz (2020) 
Dado los resultados mostrados, el investigador deduce que en las empresas bajo estudio el rol ejercido por el tesorero es vital, considerando que son pequeñas y medianas empresas, por lo cual una función clave de éste debe ser proyectar los flujos de cobros y flujos de pago esperados, tal como se ha evidenciado en las respuestas obtenidas por los encuestados, otorgándoles una alta fortaleza en este respecto.

Luego de analizados los resultados para cada uno de los indicadores que conforman la dimensión estrategias de gestión de tesorería, en la tabla 8 se reflejan los resultados alcanzados para dicha dimensión. Se observa que la misma se ubica en la categoría de muy alta aplicación $(89,02 \%)$, indicando que en estas empresas la aplicación de sus estrategias de gestión de tesorería se constituye en alta fortaleza. Así, se observa que todos los indicadores aplicados se posicionaron en el rango donde la suma de las opciones siempre y casi siempre son $75 \% \leq \mathrm{S}+\mathrm{CS}<100 \%$ indicando muy alta aplicación del indicador con alta fortaleza para las empresas, exceptuando los depósitos a plazo fijo, catalogados con baja aplicación siendo entonces una posible o leve debilidad.

Tabla 8. Dimensión: Estrategias de gestión de tesorería

\begin{tabular}{lcc}
\hline \multicolumn{1}{c}{ Indicador } & S + CS & Categorías \\
\hline Flujo de cobros & $97,73 \%$ & Muy alta aplicación / Alta fortaleza \\
Flujo de pagos & $97,73 \%$ & Muy alta aplicación / Alta fortaleza \\
Posición fecha valor & $93,18 \%$ & Muy alta aplicación / Alta fortaleza \\
Depósitos a plazo fijo & $47,73 \%$ & Baja aplicación / Leve debilidad \\
Previsiones de tesorería & $97,73 \%$ & Muy alta aplicación / Alta fortaleza \\
Funciones del tesorero & $100 \%$ & Muy alta aplicación / Alta fortaleza \\
RESUMEN DIMENSIÓN & $\mathbf{8 9 , 0 2 \%}$ & Muy alta aplicación / Alta fortaleza
\end{tabular}

Fuente: Díaz (2020)

Estos resultados son altamente coincidentes con lo manejado por Olsina (2009), quien indica que el objetivo de la gestión de tesorería es la optimización de los flujos monetarios, las previsiones de liquidez, la rentabilización de sus excedentes temporales y la gestión de las relaciones bancarias. En tesorería se toman diariamente decisiones irrevocables que afectan directamente la cuenta de resultados, en un ambiente de incertidumbre procedente tanto desde el interior de la propia empresa (decisiones estratégicas a largo plazo, política de inversión, política comercial) como del entorno en el que se desenvuelve (nivel de crédito del sistema financiero, evolución de los tipos de interés y de los tipos de cambio). 
De igual manera validan a Santoma (2008), cuando afirma que los objetivos para la gestión de tesorería, serían: impulsar y plantear la política financiera de la compañía de acuerdo con sus estrategias a largo plazo, garantizando la coherencia de los objetivos a corto plazo (presupuestos anuales) y control presupuestario de la gestión de tesorería; conseguir asegurar los flujos de entrada y salida de fondos precisos para el desarrollo de la actividad de la empresa, en el momento oportuno, con riesgos controlados y al menor coste posible; y optimizar los resultados económicos de la gestión financiera, por medio de una eficaz gestión en la obtención de recursos y colocación de excedentes.

Visto estos resultados de muy alta aplicación, a juicio del investigador, en las pequeñas y medianas empresas del sector petroquímico del municipio Miranda, las estrategias de gestión de tesorería constituyen una alta fortaleza como el conjunto de técnicas y procedimientos para hacer eficiente la administración de fondos monetarios.

En virtud de esto, las estrategias de gestión de tesorería, como parte de las estrategias financieras que desarrollan estas empresas, se perfilan hacia la administración interna de los fondos destacándose el papel de los flujos de cobro, flujos de pagos, posición fecha valor, previsiones de tesorería y funciones del tesorero; no obstante, los depósitos a plazo fijo se ubican en baja aplicación, lo cual, a criterio del investigador, puede ser efecto del entorno en el que se desenvuelven estas empresas (nivel de crédito del sistema financiero, evolución de los tipos de interés y tipos de cambio).

\section{CONCLUSIONES}

En lo que concierne al objetivo, donde se analizó las estrategias de gestión de tesorería en las pequeñas y medianas empresas del sector petroquímico, se evidencio que estas empresas presentan muy alta aplicación de las estrategias de gestión de tesorería, indicándoles una alta fortaleza en cuanto a los aspectos medidos. Se resalta que los indicadores: flujo de cobros, flujo de pagos, posición fecha valor, previsiones de tesorería y funciones del tesorero se posicionaron en muy alta aplicación; sólo los depósitos a plazo fijo ostentaron alta aplicación, siendo entonces una posible o leve debilidad.

Se concluye entonces, para el caso de las estrategias de gestión de tesorería, que aun cuando se evidencia baja aplicación de los depósitos a plazo fijo en éstas estrategias, es probable que estas empresas quieran mantener liquidez ante las condiciones de inflación que se tiene en Venezuela, obligándolas a contar con dinero a disposición para enfrentar este tipo de flagelo económico, lo cual las lleva a una baja aplicación de los depósitos a plazo fijo, para mantener liquidez inmediata.

REFRENCIAS

Brachfield, J. (2009). Gestión del crédito y cobro. Claves para prevenir la morosidad y recuperar los impagados. Editorial Profit. Barcelona

Corchero, J. y Pérez, M. (2004). Finanzas para emprendedores. Ediciones Emprende. Colombia

Delgado, J. (2010). Manual práctico de gestión de tesorería de empresas. Segunda edición. Ediciones Díaz de Santos. Bogotá, Colombia 
Fernández, M. (2006). Problemática de las Pequeñas y Mediana Empresa Venezolana. Análisis de factores interno. VI. Congreso de investigación y creación intelectuales la UNIMET. Universidad Metropolitana. Caracas

Hernández J. (2005). Las estrategias de inversión. Editorial de la Universidad de Yacambú. Barquisimeto. Venezuela

Ludevid, M. y Ollé, M. (2004). Como crear su propia empresa. Factores clave de gestión. Segunda Edición. Marcombo Boixaren Editores. España
Olsina, X. (2009). Gestión de tesorería. Profit Editorial. Barcelona, España.

Paniagua, V., Paniagua, M. y Paniagua, M. (2004). El contralor responsabilidades y funciones. 3era. Edición. Editorial Thomson. México, D.F

Santoma J. (2008). Gestión de Tesorería. Segunda edición. Ediciones Gestión 2000, S.A. Barcelona, España

Valls, J. (2003). Fundamentos de la nueva gestión de tesorería. Editorial FC. España 